\title{
Sulla dinamica relativistica di un fluido ideale conduttore di calore $\left(^{*}\right)\left({ }^{* *}\right)$.
}

\author{
Grovannt CARTnt (Messina)
}

\begin{abstract}
Sunto - Si stabiliscono le equazioni fondamentali della dinamica relativistica di un fluido ideale condultore di calore, muovendo dai principi della dinamica di un corpuscoio a massa di quiete variabile. Successivamente si deduce il tensore energetico del suddetfo mezzo che, solo nella forma, appare identico a quello dei fluidi ideali isentropici.
\end{abstract}

1. - Nella dinamica relativistica di un fluido ideale conduttore di calore attualmente esistono due punti di vista distinti.

Il primo, seguito principalmente dalla Scuola francese e da alcuni Autori italiani, fa capo al lavoro $\left({ }^{1}\right)$ di PHAM MaU QUAN ed attribuisce al tensore energetico del fluido considerato una struttura diversa da quella che ha il tensore energetico di un fluido ideale isentropico.

$\mathrm{NeI}$ secondo schema, riportato da LANDAU-LIFSHTTZ $\left(^{2}\right)$, invece il tensore energetico di un fluido ideale conduttore di calore ha la medesima forma di quello di un fluido ideale isentropico.

Ora la costruzione dei suddetti tensori energetici è fondata su certe assunzioni, che non sembrano del tutto soddisfacenti.

In conseguenza di ciò, nel presente lavoro, mi sono proposto di riesaminare la questione suaccennata alla luce dei principi della dinamica relativistica di un corpuscolo a massa di quiete variabile.

Tale riesame mi ha consentito di stabilire le equazioni fondamentali di moto di un fluido ideale relativistico conduttore di calore e di dedurre il suo tensore energetico, che risulta identico, solo nella struttura, a quello assunto da LANDAULIFSHITZ.

Le deduzioni che ho fatto utilizzano, oltre ai principi di conservazione dell'energia e dell'impulso, la concezione fisica sostenuta $\left(^{2}\right)$ validamente da LANDAU-LIFshra,

(*) Entrata in Redazione il 28 novembre 1972.

(**) Lavoro eseguito nall'ambito dall'attività dei Contratti di ricerca matematica del Consiglio Nazionale delle Ricerche.

(1) Pham Mau Quan, Ann. di Mat. pura e appl., (4), 38 (1955); Relativistic fluid Dynamies, I ciclo Bressanone, 7-16 giugno 1970, Edizioni Cremonese, Roma, 1971.

( ${ }^{2}$ L. D. Landad - E. M. Lrsfhrtz, Fluid Mechanics, Pergamon Press, 1959, pag. 505.

$\left({ }^{3}\right)$ Cfr. la cit. $\left({ }^{2}\right)$. 
secondo la quale, in base al principio di inerzia dell'energia di Einstein, ogni flusso di energia (ad es. di natura termica) implica un flusso di massa e la definizione di velocità non va connessa soltanto alla densità del flusso di massa, che si ha in assenza di conduzione termica, bensì ad una densità di flusso che tenga conto anche della corrente termica.

Per quanto concerne lo sviluppo di questo lavoro, nel n. 2 si accenna alla linea fondamentale del primo punto di vista e si pone in rilievo qualche assunzione che non sembra convincente nel primo o nel secondo schema.

Nel n. 3 dapprima si introduce la densità di massa di quiete $\varrho^{0}$, proveniente dalla distribuzione di materia e dall'energia termica, e si mostra ehe essa si comporta, durante il moto, come la cosiddetta densità "invariante " considerata $\left(^{4}\right)$ da V. Fock nel caso isentropico. Successivamente si introduce la quadridensità di corrente termica e si determina l'equazione differenziale a cui soddisfa.

Nel n. 4 si deducono, nello spazio-tempo di Minkowski, varie forme delle equazioni fondamentali della dinamica di un fluido ideale relativistico termoconduttore.

Nel n. 5 si presentano le suddette equazioni nelle usuali notazioni tridimensionali e si fa il confronto con le corrispondenti equazioni della fluidodinamica classica.

Infine nel n. 6 si deduce il tensore energetico del mezzo in esame che, come abbiamo detto, appare identico nella forma, a quello dei fluidi ideali isentropici.

Una teoria relativistica di un fluido viscoso conduttore di calore sarà esposta in un lavoro successivo.

2. - Per quanto sia a mia conoscenza, la prima formulazione di una teoria relativistica dei fluidi termodinamici con conduzione del calore è stata fatta nel 1940 da C. ECKart ( ${ }^{5}$ ). Però, nello schema proposto, la generalizzazione dell'ipotesi di Fourier risulta poco convincente perchè non utilizza l'equazione di conduzione del calore.

Per tale motivo, nel 1954, PHaMr Mau QuaN ( $\left.{ }^{\circ}\right)$ riprende la questione e propone una più completa teoria relativistica di un fluido ideale termoconduttore.

Successivamente Pichon ( $\left.{ }^{7}\right)$ integra lo schema precedente con l'aggiunta dei termini di viscosità.

Un modello di un fluido termoconduttore, diverso da quello richiamato sopra, viene presentato nel volume di LANDAU-LIFSHTTz (efr. 1. cit. $\left({ }^{2}\right)$ ).

La teoria relativistica concepita da PHanr ha le basi fisiche sulla termofluidodinamica classica che, per quanto riguarda il fenomeno della conduzione del calore, si fonda:

(4) Cfr. ad es. G. CARrni, Ann. di Mat. pura e appl., Serie IV, vol, 34 (1970).

(5) C. Eckart, Phys. Rev., 58 (1940).

(6) Cfr. la cit. (1).

(7) G. Pichon, Ann. Inst. Henri Poincaré, 2 (1965). 
a) sul fenomeno elementare della conduzione termica tradotto matematieamente dall'ipotesi di Fourier:

$$
\mathscr{J}=-\chi \operatorname{grad} \theta
$$

b) sull'equazione di conduzione del ealore

$$
-\operatorname{div} \mathscr{J}=O_{\varepsilon} \varrho \frac{d \theta}{d t}-\frac{l}{\varrho} \frac{d \varrho}{d t}
$$

Nelle (1), (2) $\mathscr{J}$ è il vettore tridimensionale densità di corrente termica, $\chi>0$ è la conducibilità termica del mezzo, $O_{v}$ il calore specifico a volume constante, $l$ il calore di dilatazione, $\theta$ la temperatura, $\varrho$ la densità di massa, $t$ il tempo.

PHaM stabilisce l'estensione relativistica dell'ipotesi di Fourier, dopo aver considerato nel sistema di istantanea quiete $K_{1}$ (quale si avrebbe anche in assenza di conduzione termica) del generico elemento fluido, le quattro quantità

$$
J_{0}^{\prime}=0, \quad J_{i}^{\prime}=-\chi \frac{\partial \theta}{\partial x^{\prime i}}
$$

che assume come le componenti del quadrivettore densità di corrente termica. Da cio segue che le componenti $J_{x}$ di tale quadrivettore, nel riferimento di osservazione, sono espresse dalla formula

$$
J_{\alpha}=-\chi \frac{\partial \theta}{\partial x^{o}}\left(g_{\alpha}^{\rho}-W^{o} W_{\alpha}\right)
$$

che estende relativisticamente l'ipotesi di Fourier.

Successivamente, con una integrazione in una regione dello spazio-tempo della Teoria della Relatività generale e con una conseguente riduzione al punto estende la (2) nella seguente relazione $\left(^{(9)}\right.$

$$
\frac{1}{c} J_{\sigma}^{\prime \sigma}=C_{v} \varrho W^{\alpha} \frac{\partial \theta}{\partial x^{\alpha}}-\frac{l}{\varrho} W^{\alpha} \frac{\partial \varrho}{\partial x^{\alpha}} .
$$

Dopo i precedenti richiami, per evitare superflue complicazioni di calcolo e cogliere meglio l'aspetto fisico delle questioni in esame, tratterò $i$ vari problemi nell'ambito della Teoria della Relatività speciale, in quanto, com'è notó, la discussione degli stessi argomenti in Relatività generale non presenta alcuna difficoltà concettuale.

(8) La (5) differisce dalla (10.4) di Pham, riportata a pag. 138 della Memoria eitata in 1. ( $\left.{ }^{(}\right)$, per il fattora $1 / 0$ a primo membro che qui figura opportunamente per ragioni dimensionali. 
Ciò posto, si indichi con $K$ il riferimento inerziale rispetto a cui si considera il moto del fluido ideale termoconduttore e si indichi con $K_{1}$ il sistema di istantanea quiete del generico elemento $M$ del mezzo.

Se supponiamo di utilizzare delle coordinate galileiane, introdotte in $K$, la metrica dello spazio-tempo corrispondente assume la seguente forma:

$$
d s^{2}=\left(d x^{0}\right)^{2}-\left(d x^{1}\right)^{2}-\left(d x^{2}\right)^{2}-\left(d x^{3}\right)^{2}=g_{\mu \nu} d x^{\mu} d x^{\nu}
$$

dove $\left({ }^{9}\right)$ si è posto $x^{0}=c t$, essendo $t$ il tempo e $o$ la velocità della luce in ogni sistema inerziale di riferimento; $x^{1}, x^{2}, x^{3}$ sono coordinate cartesiane ortogonali. Si ha inoltre: $g_{\mu \nu}=1$ per $\mu=\nu=0 ; g_{\mu \nu}=-1$ per $\mu=\nu=1,2,3 ; g_{\mu \nu}=0$ per $\mu \neq \nu$.

La velocità cronotopica del generico elemento materiale $M \equiv\left(x^{\sigma}\right)$ è definita da

$$
W^{\sigma}=\frac{d x^{\alpha}}{d s}=\left(\alpha, \frac{\alpha}{c} v\right)
$$

essendo $\alpha=\left(1-v^{2} / c^{2}\right)^{-\frac{1}{2}}, \boldsymbol{v}(M, t)$ la velocità ordinaria tridimensionale rispetto a $K$.

Ciò premesso, notiamo che la (5), scritta in $K_{1}$ con le notazioni tridimensionali, assume la seguente forma:

$$
O_{v} \varrho_{1} \frac{d \theta}{d t_{1}}-\frac{l}{\varrho_{1}} \frac{d \varrho_{1}}{d t_{1}}=-\operatorname{div} \mathscr{J}_{1}
$$

essendo $c \bar{W}^{\sigma}\left(\partial \theta / \partial \bar{x}^{\sigma}\right)=d \theta / d t_{1}$ ed avendo indicato con $\bar{x}^{\sigma}, \varrho_{1}, t_{1}, \mathscr{J}_{1}$ rispettivamente le coordinate di $M$, la densità di massa, il tempo ed il vettore tridimensionale densità di corrente termica, tutti valutati in $K_{1}$ e con $\bar{W}^{\sigma}=d \bar{x}^{\sigma} / d s$ la quadrivelocità di $M$ in $K_{1}$.

D'altra parte, se si utilizza il principio d'inerzia dell'energia di Einstein, l'equazione che esprime la variabilità della massa di quiete di $M$ rispetto a $K_{1}$ è ovviamente:

$$
\frac{\partial}{\partial t_{1}}\left(\varrho_{1} d V_{1}\right)=\frac{d}{d t_{1}}\left(\varrho_{1} d V_{1}\right)=\frac{1}{c^{2}} \int_{\Sigma} \mathscr{J}_{1 n} d \sigma=-\frac{1}{a^{2}} \operatorname{div} \mathscr{J}_{1} d V_{1}
$$

da cui, in virtù della ben nota relazione cinematica

$$
\frac{\partial}{\partial t_{1}}\left(d V_{1}\right)=\frac{d}{d t_{1}}\left(d V_{1}\right)=\operatorname{div} v_{1} d V_{1}
$$

si trae

$$
c^{2} \frac{d \varrho_{1}}{d t_{1}}+c^{2} \varrho_{1} \operatorname{div} v_{1}=-\operatorname{div} \mathscr{J}_{1}
$$

${ }^{(9)}$ Gli indici in basso sono di covarianza, quelli in alto di contravarianza ed è sottintesa ogni sommatoria che satura un indice di covarianza con uno di contravarianza. Inoltre gli indici greci assumono i valori $0,1,2,3$; quelli latini 1, 2, 3 . 
Nelle (9), (10) con $d V_{1}$ si è indicato il volume di quiete dell'elemento materiale $M$ e con $\Sigma$ la sua superficie.

Ora, le definizioni del calore specifico a volume costante, del calore di dilatazione del fluido, l'equazione di continuità ed il suddetto principio d'inerzia dell'energia di Einstein consentono di serivere le seguenti relazioni

$$
\begin{aligned}
& \varrho_{1} O_{v} d \theta=e^{2} d \varrho_{1}, \\
& l \frac{d\left(1 / \varrho_{1}\right)}{d t_{1}}=-\frac{l}{\left(\varrho_{1}\right)^{2}} \frac{d \varrho_{1}}{d t_{1}}=c^{2} \operatorname{div} v_{1}
\end{aligned}
$$

e di rilevare quindi l'identità delle due equazioni (8) e (11).

Dobbiamo osservare però che nel passaggio dalla (9) alla (11) (equivalente alla (8)) si è ammesso tacitamente che il flusso di calore per conduzione non modifica la velocità $v_{1}$ dell'elemento $M$ rispetto a $K_{1}$.

In effetti, questa circostanza si presenta nella termofluidodinamica relativistica quando non ha rilevanza fisica la propagazione del calore per conduzione.

Nel caso in esame, invece, la propagazione del calore per conduzione imprime all'elemento $M$ un impulso (e quindi una velocità $v_{1}^{*} \neq 0$ ) rispetto a $K_{1}$, per cui la sua velocità rispetto a $K_{1}$ sarà

$$
\frac{d \bar{x}^{i}}{d t_{1}}=v_{1}^{i}+v_{1}^{* i}=v_{1}^{* i} \neq 0
$$

Pertanto, in $K_{1}$, le derivate locali delle varie grandezze non si identificano più con le corrispondenti derivate sostanziali, come risulta evidente, ad es., da

$$
\frac{\partial}{\partial t_{1}}\left(d V_{1}\right)=\frac{d}{d t_{1}}\left(d V_{1}\right)-v_{1}^{* i} \frac{\partial}{\partial \bar{x}^{i}}\left(d \nabla_{1}\right)
$$

In conseguenza di ciò, nel caso in esame, non valgono più le (9), (10), (13), (8), (5), sull'ultima delle quali è impostata sostanzialmente la teoria relativistica seguita dalla Scuola francese.

D'altra parte nella trattazione riportata da LANDAU-LIFSHITz (cfr. pp. 505-506 del vol. cit. 1. $\left(^{2}\right)$ ) l'assunzione fatta per il vettore densità di flusso particellare $n_{\sigma}=n W_{\sigma}+v_{\sigma}$, l'equazione a cui esso soddisfa e la richiesta d'aumento dell'entropia per il generico elemento del mezzo non sembrano convincenti.

Pertanto nei numeri seguenti, riesamineremo tatta la questione utilizzando i principi di conservazione dell'energia e dell'impulso, i principi della dinamica di un corpuscolo a massa di quiete variabile e terremo conto della circostanza che la definizione di velocità non va connessa soltanto al flusso di massa, bensì ad una densità di flusso che implichi anche la corrente termica. 
3. - Dopo le precedenti considerazioni si introduca oltre a $K_{1}$ (sistema di istantanea quiete dell'emento $M, d m_{1}$, in assenza di conduzione termica) il riferimento inerziale $K^{\prime}$ di istantanea quiete dell'elemento totale $N, d m_{0}$, costituito dalla massa $d m_{1}^{0}$ proveniente da $d m_{1}$ e dalla massa $d m_{r}^{0}$ di origine termica, che esiste certamente in virtù del principio d'inerzia dell'energia di Einstein.

Si indichi con $v^{\prime}=0$ la velocità di $N, d m_{0}$ rispetto a $K^{\prime}$ e con $v$ la velocità ordinaria di $K^{\prime}$ rispetto al sistema di osservazione $K$.

Si denoti inoltre con $\boldsymbol{v}_{1}$ la velocità di $M\left(\right.$ o di $\left.K_{1}\right)$ rispetto a $K^{\prime}$, con $d m_{2}, \boldsymbol{v}_{2}$ e $\mathscr{J}^{\prime}$ rispettivamente la massa di quiete proveniente dall'energia termica di conduzione, la sua velocità ordinaria e la densità di corrente termica, escendo $\boldsymbol{v}_{2}$ ed $\mathscr{J}^{\prime}$ valutate rispetto a $K^{\prime}$.

Allora, le energie $d \mathscr{E}_{0}, d \mathscr{E}_{1}^{\mathscr{0}}$ rispettivamente dell'elemento $N, d m_{0}$ ed $M, d m_{1}$, la energia termica di conduzione $d \mathscr{E}_{T}^{0}$ contenuta in $d m_{0}$, i corrispondenti impulsi $d \boldsymbol{q}^{\prime}$, $\boldsymbol{d} \boldsymbol{q}_{1}^{0}, \boldsymbol{d \boldsymbol { q } _ { T } ^ { 0 }}$ avranno in $\boldsymbol{K}^{t}$ ovviamente le seguenti espressioni

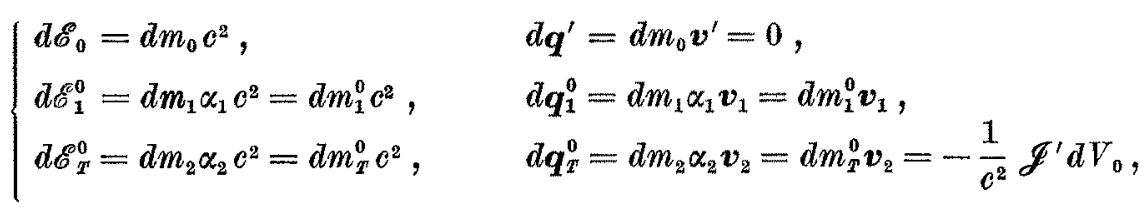

dove si è posto $\alpha_{1}=\left(1-v_{1}^{2} / c^{2}\right)^{-\frac{1}{2}}, \alpha_{2}=\left(1-v_{2}^{2} / c^{2}\right)^{-\frac{1}{2}}$ e si sono indicate con $d m_{1}^{0}=$ $=d m_{1} \alpha_{1}$ e $d m_{m}^{0}=d m_{2} \alpha_{2}$ rispettivamente la massa di $M$ e quella di origine termica, entrambe valutate rispetto a $K^{\prime}$, ed inoltre si è denotato con $d V_{0}$ il volume dell'elemento $N$ in $K^{\prime}$.

I due principi di conservazione dell'energia e dell'impulso, scritti in $K^{\prime}$, ci forniscono

$$
\left\{\begin{array}{l}
d \mathscr{E}_{0}=d \mathscr{E}_{1}^{0}+d \mathscr{E}_{r}^{0} \\
d \boldsymbol{q}^{\prime}=d \boldsymbol{q}_{1}^{0}+d \boldsymbol{q}_{2}^{0}
\end{array}\right.
$$

da cui segue in virtù delle (16):

$$
\left\{\begin{array}{l}
d m_{0}=\alpha_{1} d m_{1}+\alpha_{2} d m_{2}=d m_{1}^{0}+d m_{T}^{0} \\
d m_{0} \boldsymbol{v}^{\prime}=d m_{1}^{0} \boldsymbol{v}_{1}+d m_{T}^{0} \boldsymbol{v}_{2}=d m_{1}^{0} \boldsymbol{v}_{1}-\frac{1}{c^{2}} \mathscr{J}^{\prime} d \boldsymbol{V}_{0}
\end{array}\right.
$$

Se ora si introducono le varie densità rispetto a $K^{\prime}$ e cioè:

$$
d m_{0}=\varrho^{0} d V_{0}, \quad d m_{1}^{0}=\varrho_{1}^{0} d V_{0}, \quad d m_{t}^{0}=\varrho_{r}^{0} d V_{0}
$$

dalle (18) si ricava

$$
\left\{\begin{array}{l}
\varrho^{0}=\varrho_{1}^{0}+\varrho_{T}^{0} \\
\varrho^{0} v^{\prime}=\varrho_{1}^{0} v_{1}-\frac{1}{c^{2}} \mathscr{J}^{\prime}
\end{array}\right.
$$


Dopo di ciò, se si riflette che l'elemento totale $N, d m_{0}$ ha nulla la velocità $\boldsymbol{v}^{\prime}$ rispetto a $K^{\prime}$ e che pertanto risulta nullo il flusso dell'impulso totale attraverso la superficie $\Sigma$ di $N, d m_{0}$, si ha:

$$
\frac{\partial}{\partial t^{\prime}}\left(\varrho^{0} d V_{0}\right)=\frac{d}{d t^{\prime}}\left(\varrho^{0} d V_{0}\right)=\int_{\Sigma} \varrho^{0} v_{n}^{\prime} d \sigma=\int_{\Sigma}\left(\varrho_{0} \boldsymbol{v}_{1}-\frac{1}{c^{2}} \mathscr{J}^{\prime}\right)_{n} d \sigma=0
$$

dove $t^{\prime}$ è il tempo valutato in $K^{\prime}$.

Pertanto in $K^{\prime}$ vale la seguente relazione

$$
\frac{d\left(d m_{0}\right)}{d t^{\prime}}=\frac{d}{d t^{\prime}}\left(\varrho^{0} d V_{0}\right)=0 .
$$

La utilizzazione della (10), applicata a $d V_{0}$, consente di dedurre dalla $\left(20^{\prime}\right)$ la seguente equazione:

$$
\frac{d \varrho^{0}}{d t^{\prime}}+\varrho^{0} \operatorname{div} v^{\prime}=\frac{\partial \varrho^{0}}{\partial t^{\prime}}+\operatorname{div}\left(\varrho^{0} v^{\prime}\right)=0
$$

Poichè in $K^{\prime}$ si ha:

$$
\boldsymbol{v}^{\prime}=0, \quad \operatorname{div} \boldsymbol{v}^{\prime}=\frac{\partial v^{\prime i}}{\partial x^{\prime i}} \neq 0, \quad W^{\prime}=\left(1, \frac{\boldsymbol{v}^{\prime}}{c}\right)
$$

la (21) può porsi agevolmente nella seguente forma covariante

$$
\frac{\partial}{\partial x^{\prime \sigma}}\left(\varrho^{0} W^{\prime \sigma}\right)=0
$$

figurando, a primo membro, un invariante relativistico.

Di conseguenza la (23) nel riferimento $K$ diventa

$$
\frac{\partial}{\partial x^{\sigma}}\left(\varrho^{0} W^{\sigma}\right)=0
$$

Notiamo ancora che nella $\left(\mathbf{1}_{\mathbf{2}}\right)$, si è posto,

$$
d m_{2}^{0} \boldsymbol{v}_{\mathbf{2}}=-\frac{1}{e^{2}} \mathscr{J}^{\prime} d V_{0}
$$

da eui segue ovviamente

$$
\varrho_{x}^{0}=\frac{\mathscr{J}^{\prime}}{c^{2} v_{2}}
$$

Inoltre dalla $\left(20^{\prime}\right)$, in virtù della $\left(18_{1}\right)$, segue:

$$
\frac{d}{d t^{\prime}}\left(d m_{1}^{0}\right)=-\frac{d}{d t^{\prime}}\left(d m_{x}^{0}\right)=-\frac{\partial}{\partial t^{\prime}}\left(d m_{r}^{0}\right) .
$$


Poichè l'elemento $N, d m_{0}$ ha velocità istantanea nulla rispetto a $K^{\prime}$, al vettore $\mathscr{J}^{\prime}$ puo applicarsi il teorema della divergenza nella forma usuale, come abbiamo fatto nella (9). Allora, per quanto riguarda la variazione, per unità di tempo, dell'energia termica contenuta in $N, d V_{0}$, si ha:

$$
\frac{\partial}{\partial t^{\prime}}\left(d m_{T}^{0}\right)=\frac{\partial}{\partial t^{\prime}}\left(\varrho_{T}^{0} d V_{0}\right)=\frac{1}{c^{2}} \int_{\Sigma} \mathscr{J}_{n}^{\prime} d \sigma=-\frac{1}{c^{2}} \operatorname{div} \mathscr{F}^{\prime} d V_{0},
$$

da cui, in virtù delle $(10),(22)$, segue:

$$
\frac{\partial\left(U_{T}^{0} W^{\prime 0}\right)}{\partial x^{\prime 0}}+\frac{\partial}{\partial x^{\prime i}}\left(U_{T^{i}}^{0} W^{\prime i}+\frac{1}{c} \mathscr{J}_{i}^{\prime}\right)=0
$$

essendo $V_{x^{*}}^{0}=0^{2} \varrho_{x}^{0}$ l'energia termica per unità di volume.

Se ora si pone

$$
T^{\prime \sigma}=U_{T}^{0} W^{\prime \sigma}+J^{\prime \sigma}=c^{2} \varrho_{T}^{0} W^{\prime \sigma}+J^{\prime \sigma}
$$

essendo

$$
J^{\prime 0}=0, \quad J^{\prime i}=\frac{1}{c} \mathscr{J}_{i}^{\prime}
$$

la (27) assume la seguente forma

$$
\frac{\partial \Gamma^{\prime \sigma}}{\partial x^{\prime \sigma}}=\Gamma_{l \sigma}^{\prime \sigma}=0
$$

che dev'essere covariante rispetto alle trasformazioni di Lorentz perchè traduce matematicamente un principio fisico di conservazione.

Da ciò segue che le $\Gamma^{\text {ya }}$ sono le componenti contravarianti di un quadrivettore, che puo denotarsi la quadridensitd di corrente termica.

Pertanto, nel riferimento $K$, la $\left(27^{\prime}\right)$ si muta nell'equazione

$$
\frac{\partial \Gamma^{\sigma}}{\partial x^{\sigma}}=\Gamma_{/ \sigma}^{\sigma}=0
$$

essendo

$$
\Gamma^{\sigma}=U_{x}^{0} W^{\sigma}+J^{\sigma}=c^{2} \varrho_{x}^{0} W^{\sigma}+J^{\sigma}
$$

Poichè $U_{T}^{0}$ è un invariante relativistico (come $H_{0}, \varrho^{0}$, ecc.), $U_{T}^{0} W^{\sigma}$ è un quadrivettore che possiamo designare la quadridensità di corrente termica di convezione. Allora dalle (28), (31) segue ovviamente la natura tensoriale di $J^{\sigma}$ che chiameremo la quadridensità di corrente termica di conduzione.

Da quanto è stato esposto possiamo dire che la quadridensità di corrente termica $\Gamma^{\sigma}$ si compone della quadridensità di corrente termica di convezione $U_{r}^{0} W^{\sigma} \mathrm{e}$ di quella di conduzione $J^{\sigma}$. 
Le componenti di $J^{\sigma}$ si ottengono da quelle di $J^{\prime \sigma}$ con le seguenti note formule

$$
\left\{\begin{array}{l}
J^{0}=\alpha\left(J^{\prime 0}+\frac{1}{e} v_{i} J^{\prime i}\right), \\
J^{i}=J^{\prime i}+\frac{\alpha}{e} J^{\prime 0} v_{i}+(\alpha-1)\left(v_{i} J^{\prime i}\right) \frac{v_{i}}{v^{2}} .
\end{array}\right.
$$

Poichè si ha

$$
\frac{\partial J^{\prime \mu}}{\partial x^{\prime \mu}}=\frac{\partial J^{\sigma}}{\partial x^{\sigma}}
$$

ossia

$$
\operatorname{div} \mathscr{J}^{\prime}=\frac{1}{e^{2}} \frac{\partial}{\partial t}\left(\alpha v \cdot \mathscr{J}^{\prime}\right)+\operatorname{div} \mathscr{J}
$$

ne segue, tenendo conto delle (16) e (25) che in prima approssimazione vale la relazione $(*)$

$$
\operatorname{div} \mathscr{J} \simeq \operatorname{div} \mathscr{J}^{\prime}
$$

Infine dalle (21), $\left(19_{1}\right)$ si desume agevolmente la relazione

$$
c^{2}\left[\frac{\partial \varrho_{1}^{0}}{\partial t^{\prime}}+\operatorname{div}\left(\varrho_{1}^{0} v_{1}\right)+\frac{\partial \varrho_{r}^{0}}{\partial t^{\prime}}\right]=\operatorname{div} \mathscr{J}^{\prime}
$$

che, nel caso in cui il gradiente della temperatura del mezzo $T_{0}$ (valutata rispetto a $K^{\prime}$ ) non è molto grande ossia quando vale la nota legge di Fourier (1), assume la seguente forma

$$
o^{2}\left[\frac{\partial \varrho_{1}^{0}}{\partial t^{\prime}}+\operatorname{div}\left(\varrho_{1}^{0} v_{1}\right)+\frac{\partial \varrho_{r}^{0}}{\partial t^{\prime}}\right]=-\operatorname{div}\left(\chi \operatorname{grad} T_{0}\right)
$$

Chiudiamo questo numero rilevando che la densità di massa di quiete $\varrho^{\natural}$, proveniente dalla distribuzione di materia e dall'energia termica di conduzione, si comporta, durante il moto, come la cosiddetta densità "invariante" considerata da V. Fock nel caso in cui $\left({ }^{10}\right)$ si prescinda dalla conduzione del calore.

La (24) esprime, rispetto a $K$, la conservazione della massa di quiete $d m_{0}$ del generico elemento $N$.

Si può desumere agevolmente (ad es. muovendo dalla $\left(20^{\prime}\right)$ da cui proviene la (24)) che la suddetta conservazione con le notazioni tridimensionali si esprime nel seguente modo:

$$
\frac{d}{d t}\left(d m_{0}\right)=0
$$

$\left(^{*}\right)$ Infatti dalle (16) e (25) si desume che $\mathscr{J}$ è dello stesso ordine di $v$; pertanto sviluppando in potenze di $v / c$ e trattenendo i termini di primo ordine, segue la (33).

(10) Cfr. ad es. 1. cit. $\left({ }^{4}\right)$. 
4. - Allo scopo di dedurre le equazioni fondamentali della dinamica relativistica di un fluido ideale termoconduttore ci limitiamo a considerare il caso più semplice, ossia il caso in cui sono assenti le forze di massa (di natura esterna).

Se si indica con $d S_{m}$ l'entropia relativistica della generica particella $N$ del fluido rispetto a $K^{\prime}$ e con $\bar{S}_{0}$ l'entropia relativistica, per unità di massa "invariante ", si ha:

$$
d S_{m}=\bar{S}_{0} d m_{0} .
$$

Poichè è nullo il flusso dell'impulso totale attraverso la superficie $\Sigma$ dell'elemento $N$ ed il processo fisico che interessa l'elemento $N$, $a m_{a}$, nel riferimento $K^{\prime}$ viene ad essere puramente adiabatico, in virtù della $\left(20^{\prime}\right)$, si può scrivere:

$$
\frac{d}{d t^{\prime}}\left(d S_{m}\right)=\frac{d \bar{S}_{0}}{d t^{\prime}} d m_{0}=\mathbf{0}
$$

Dopo di cio, si denoti con $d \bar{m}_{0}$ la massa totale di quiete dell'elemento $N$ rispetto a $K^{\prime}$ e con $d E_{0}$ la sua energia totale di quiete.

In virtù del principio d'inerzia dell'energia si ha

$$
d \bar{m}_{0}=\frac{d E_{0}}{c^{2}}=\frac{1}{c^{2}}\left(d E_{o}^{(i)}+p^{\prime} d V_{0}\right)
$$

dove $d E_{0}^{(i)}=U_{0} d V_{0}$ è l'energia interna relativistica di $N, U_{0}$ l'energia interna per unità di volume e $p^{\prime} d V_{0}$ è l'energia ( $\left.{ }^{11}\right)$ di quiete conferita ad $N$ dalle forze di pressione.

Se si considera ora l'entalpia $H_{0}=U_{0}+p^{\prime}$, per unità di volume, dalla (39) segue

$$
d \bar{m}_{0}=\frac{1}{c^{2}}\left(U_{0}+p^{\prime}\right) d V_{0}=\frac{1}{c^{2}} H_{0} d V_{0}=\frac{\bar{H}_{0} d m_{0}}{c^{2}}
$$

dove $\bar{H}_{0}=H_{0} / \varrho^{0}$ è l'entalpia per unità di massa.

Allora dalle $\left(20^{\prime}\right),(40)$ si trae

$$
\frac{d}{d t^{\prime}}\left(d \bar{m}_{0}\right)=\frac{d}{d t^{\prime}}\left(\frac{\bar{H}_{0} d m_{0}}{c^{2}}\right)=\frac{1}{e^{2}} \frac{d \bar{H}_{0}}{d t^{\prime}} d m_{0}
$$

che, in virtù della (38) e della ben nota relazione termodinamica

$$
d \bar{H}_{0}=T_{0} d \bar{S}_{0}+\frac{1}{\varrho_{0}} d p^{\prime}
$$

ci fornisee

$$
\frac{d}{d t^{\prime}}\left(d m_{0}\right)=\frac{1}{c^{2}} \frac{\partial p^{\prime}}{\partial t^{\prime}} d V_{0}
$$

(11) Cfr. 1. cit. $\left({ }^{4}\right)$. 
poichè in $K^{\prime}$ si ha:

$$
\frac{\partial}{\partial t^{\prime}}=\frac{d}{d t^{\prime}}
$$

Dopo le precedenti considerazioni, le equazioni relativistiche della dinamica dei fluidi ideali, in presenza del processo dissipativo della conduzione del calore ed in assenza di forze di massa, si scrivono agevolmente.

Infatti, nel riferimento $K^{\prime}$, l'equazione esprimente la variazione per unità di tempo della massa totale di quiete dell'elemento $N$ e la generica equazione tridimensionale dell'impulso sono:

$$
\left\{\begin{array}{l}
\frac{d}{d t^{\prime}}\left(d \bar{m}_{0}\right)=\frac{1}{c^{2}} \frac{\partial p^{\prime}}{\partial t^{\prime}} d V_{0} \\
\frac{d}{d t^{\prime}}\left(d \bar{m}_{0} v_{i}^{\prime}\right)=-\frac{\partial p^{\prime}}{\partial x^{\prime i}} d V_{0},
\end{array}\right.
$$

che, in virtù della $\left(22_{3}\right)$, possono porsi nella seguente forma:

$$
\left\{\begin{array}{l}
\frac{d}{d s^{\prime}}\left(d \bar{m}_{0} W^{\prime 0}\right)=\frac{1}{c^{2}} \frac{\partial p^{\prime}}{\partial x^{\prime 0}} d V_{0}, \\
\frac{d}{d s^{\prime}}\left(d \bar{m}_{0} W^{\prime i}\right)=-\frac{1}{c^{2}} \frac{\partial p^{\prime}}{\partial x^{\prime i}} d V_{0}
\end{array}\right.
$$

Se ora si osserva che, in conseguenza del carattere d'invarianza $\left({ }^{12}\right)$ relativistica di $p^{\prime}$, le quattro quantità

$$
f_{\sigma}^{\prime}=\frac{1}{e^{2}} \frac{\partial p^{\prime}}{\partial x^{\prime \sigma}}
$$

sono le componenti covarianti di un quadrivettore (con riferimento alla metrica $(6)$ ) le cui componenti contravarianti sono

$$
f^{\prime \sigma}=\frac{1}{c^{2}} g^{\sigma \nu} \frac{\partial p^{\prime}}{\partial x^{\prime \nu}}=\frac{1}{c^{2}}\left(\frac{\partial p^{\prime}}{\partial x^{\prime 0}},-\frac{\partial p^{\prime}}{\partial x^{\prime i}}\right)
$$

si riconosce immediatamente che le (44) sono rispettivamente l'equazione temporale e le equazioni spaziali che si traggono dalla seguente equazione quadridimensionale

$$
\frac{d}{d s^{\prime}}\left(d \bar{m}_{0} W^{\prime} \sigma\right)=f^{\prime} \sigma V_{0}
$$

$\left(^{12}\right)$ Cfr. 1. eit. $\left({ }^{4}\right)$. 
Nel riferimento $K$, la (47) si muta evidentemente nella seguente equazione

$$
\frac{d}{d s}\left(d \bar{m}_{0} W^{\sigma}\right)=f^{\sigma} d V_{0},
$$

essendo

$$
f^{\sigma}=\frac{1}{c^{2}} g^{\sigma \nu} \frac{\partial p}{\partial x^{v}}=\frac{1}{c^{2}}\left(\frac{\partial p}{\partial x^{0}},-\frac{\partial p}{\partial x^{i}}\right)
$$

la quadridensità di forza di Minkowski agente su $N$.

Sulla (48), com'è noto, si fonda la dinamica relativistica di un corpuscolo a massa di quiete variabile; essa può presentarsi in varie forme. Così, se si utilizza la ben nota relazione $\left({ }^{13}\right)$ :

$$
\frac{d}{d s}\left(d V_{0}\right)=\frac{\partial W^{v}}{\partial x^{v}} d V_{0}
$$

e si pone $d \bar{m}_{0}=\varrho_{0} d V_{0}$, dalla (48) si trae la seguente relazione

$$
\frac{d}{d s}\left(\varrho_{0} W^{\sigma}\right)+\varrho_{0} \frac{\partial W^{v}}{\partial x^{\nu}} W^{\sigma}=\frac{1}{0^{2}} g^{\sigma \nu} \frac{\partial p}{\partial x^{\nu}}
$$

che compendia quattro equazioni indipendenti di moto, corrispondenti ai valori $0,1,2,3$ di $\sigma$.

Invece, se si sviluppa il primo membro della (48), scrivendola nel seguente modo

$$
\varrho_{0} d V_{0} \frac{d W^{\alpha}}{d s}+W^{\sigma} \frac{d\left(d \bar{m}_{0}\right)}{d s}=f^{\alpha} d V_{0}
$$

e si tiene conto della relazione

$$
\frac{d}{d s}\left(d \bar{m}_{0}\right)=\frac{1}{a^{2}} \frac{d p}{d s} d V_{0},
$$

che si trae agevolmente dalla $(42)$, dalla $\left(48^{\prime}\right)$ segue

$$
\varrho_{0} \frac{d W^{\sigma}}{d s}=\frac{1}{c^{2}} g^{\sigma y} \frac{\partial p}{\partial x^{y}}-\frac{1}{c^{2}} \frac{d p}{d s} W^{\sigma} .
$$

Ma, poichè sussistono le seguenti relazioni

$$
W_{\sigma} W^{\sigma}=1, \quad W_{\sigma} \frac{\partial W^{\sigma}}{\partial x^{\nu}}=0, \quad \frac{d p}{d s}=\frac{\partial p}{\partial x^{\sigma}} W^{\sigma},
$$

si verifica agevolmente che la composizione tensoriale di ambo i membri della (53) con $W_{\sigma}$ ei fornisce una identità.

(13) G. CaRINI, Rend. Ist. Lomb., A 102 (1968). 
Allora l'equazione che si ottiene dalla (53) per $\sigma=0$ è una conseguenza delle tre equazioni che si hanno per $\sigma=1,2,3$.

Ne segue che per avere quattro equazioni differenziali di moto indipendenti occorre considerare insieme alla (53) la seguente relazione

$$
\frac{\partial}{\partial x^{p}}\left(\varrho_{0} W^{v}\right)=\frac{1}{e^{2}} \frac{d p}{d s}
$$

che si desume agevolmente dalla (52) dopo aver utilizzato la (50).

Pertanto il sistema che ne risulta è il seguente

$$
\left\{\begin{array}{l}
\frac{\partial}{\partial x^{\nu}}\left(\varrho_{0} W^{\nu}\right)=\frac{1}{c^{2}} \frac{d p}{d s} \\
\varrho_{0} \frac{d W^{\sigma}}{d s}=\frac{1}{e^{2}} g^{\sigma v} \frac{\partial p}{\partial x^{\nu}}-\frac{1}{c^{2}} \frac{d p}{d s} W^{\sigma} .
\end{array}\right.
$$

Fin qui si è supposto tacitamente che il fluido in esame sia, termodinamicamente parlando, a due parametri.

Com'è ben noto ( $\left.{ }^{14}\right)$, quando il suo stato fisico è caratterizzato dai valori di $p$ e di $\varrho^{0}$, la natura del mezzo stesso è determinata qualora siano note le tre relazioni funzionali:

$$
T_{0}=T_{0}\left(p, \varrho^{0}\right) ; \quad \bar{S}_{0}=\bar{S}_{0}\left(p, \varrho^{0}\right) ; \quad \bar{U}_{0}=\bar{U}_{0}\left(p, \varrho^{0}\right)
$$

dette "equazioni di stato" del sistema.

Allora, poichè in $K^{\prime}$ il processo è, come abbiamo visto, adiabatico, la quinta equazione che completa le quattro equazioni (51) o il sistema (56) è la seguente:

$$
d \bar{U}_{0}+p d \frac{1}{\varrho_{0}}=0
$$

che si desume, nelle ipotesi presupposte, dalla nota relazione termodinamica

$$
T_{0} d \bar{S}_{0}=d \bar{U}_{0}+p d \frac{1}{\varrho_{0}}
$$

5. - Per trascrivere con le notazioni tridimensionali le quattro equazioni differenziali di moto che si traggono dalla (48) è utile osservare che si ha:

$$
\frac{d}{d s}=\frac{\alpha}{c} \frac{d}{d t}, \quad d m=\frac{d \bar{m}_{0}}{\sqrt{1-\beta^{2}}}=\alpha d \bar{m}_{0}, \quad \alpha d V=d V_{0},
$$

(14) Cfr. ad as. L. I. SEDov, Introduction to the Mechanics of a Continuous Medium, AddisonWasley Publishing Company, Inc. 1965, pag. 156 e segg. 
essendo $d m$ e $d V$ rispettivamente la massa totale di moto ed il volume dell'elemento $N$ rispetto a $K$.

Dopo di ciò, dalla (48) si deducono le seguenti equazioni

$$
\left\{\begin{array}{l}
\frac{d}{d t}(d m)=\frac{1}{e^{2}} \frac{\partial p}{\partial t} d V \\
\frac{d}{d t}(d m v)=-\operatorname{grad} p d V,
\end{array}\right.
$$

da cui ne emerge chiaramente l'interpretazione fisica.

Infatti la $\left(61_{1}\right)$ esprime la legge di variazione col tempo della massa totale du moto di $N$; la sua densità di sorgente è (in assenza di forze di massa) $\left(1 / c^{2}\right)(\partial p / \partial t)$. La $\left(61_{2}\right)$ è l'equazione vettoriale tridimensionale che prende il posto della classica equazione di Newton nell'ambito della fluidodinamica relativistica per un fluido ideale termoconduttore.

Si noti però che $d m$, diversamente da quanto accade quando si è in assenza della conduzione di calore, include anche la massa proveniente (in virtù del principio di inerzia dell'energia) dal calore ricevuto da $N$ per conduzione.

Se ora si introducono la densità totale di massa di moto cioè

$$
\varrho=\frac{d m}{d V}
$$

dell'elemento $N$ rispetto a $K$ e si tien presente la nota relazione cinematica

$$
\frac{d}{d t}(d V)=\operatorname{div} v d V
$$

dalle (61) si traggono le seguenti equazioni

$$
\left\{\begin{array}{l}
\frac{d \varrho}{d t}+\varrho \operatorname{div} v=\frac{1}{c^{2}} \frac{\partial p}{\partial t} \\
\varrho \frac{d v}{d t}=-\operatorname{grad} p-\frac{1}{c^{2}} \frac{\partial p}{\partial t} v
\end{array}\right.
$$

che generalizzano al caso in esame l'ordinaria equazione di continuità e la classica equazione vettoriale di Eulero.

Infine, indicando con

$$
h=\varrho c^{2}=\varrho_{0} \alpha^{2} c^{2}
$$

la densità di energia (di moto) di $N$ rispetto a $K$ e tenendo presente la relazione

$$
\frac{d \varrho}{d t}+\varrho \operatorname{div} v \equiv \frac{\partial \varrho}{\partial t}+\operatorname{div}(\varrho v)
$$


le (64) possono porsi nella seguente forma:

$$
\left\{\begin{array}{l}
\frac{\partial h}{\partial t}+\operatorname{div}(h v)=\frac{\partial p}{\partial t} \\
\frac{\partial}{\partial t}\left(\varrho v_{i}\right)+\frac{\partial}{\partial x^{k}}\left(p \delta_{i k}+\varrho v_{i} v_{k}\right)=0,
\end{array}\right.
$$

suscettibile di una interpretazione fisica diversa da quella data precedentemente. (Nelle (66) $\delta_{i k}$ è il tensore di Kronecker).

Per capire la suddetta interpretazione fisica, è utile osservare che le (66), nel caso dei moti lenti del fluido in esame, ci forniscono le equazioni della fluidodinamica classica che regolano il flusso dell'energia ed il flusso della quantità di moto nel mezzo considerato.

Allo scopo di dedurre cio, si tenga presente che si ha:

$$
h=\alpha^{2} c^{2} \varrho_{0}=\alpha^{2} H_{0}=\alpha^{2}\left(U_{0}+p\right) .
$$

Se ora si indica con $U_{0}^{*}$ la densità di energia interna di quiete (per unità di volume) rispetto a $K$, si può scrivere:

$$
U_{0}^{*}=\frac{d E_{0}^{(i)}}{d V}=\frac{U_{0} d V_{0}}{d V}=U_{0} \alpha=\frac{c^{2} \varrho_{0}+U_{p}}{\sqrt{1-\beta^{2}}}=c^{2} \varrho^{*}+U_{c}
$$

dove $U_{p} \grave{e}$ la densità di energia interna non relativistica rispetto a $K^{\prime}, V_{c}=\alpha U_{p}$ l'ordinaria energia interna rispetto a $K \mathrm{e}$

$$
\varrho^{*}=\frac{d m_{0}}{d V}=\varrho^{0} \alpha=\left(\varrho_{1}^{0}+\varrho_{r}^{0}\right) \alpha \simeq \varrho_{1}^{0}
$$

l'ordinaria densità di massa non relativistica rispetto a $K$.

Dopo di ciò dalla (67) segue:

$$
h=\frac{c^{2} \varrho^{*}+U_{c}}{\sqrt{1-\beta^{2}}}+\frac{p}{1-\beta^{2}} \simeq c^{2} \varrho^{*}+U_{c}+p+\frac{1}{2} \varrho^{*} v^{2}
$$

In conseguenza la $\left(66_{1}\right)$ si muta nella seguente equazione

$$
\frac{\partial}{\partial t}\left(U_{\circ}+\frac{1}{2} \varrho^{*} v^{2}\right)+\operatorname{div}\left[\left(H_{c}+\frac{1}{2} \varrho^{*} v^{2}\right) v\right]+c^{2}\left[\frac{\partial \varrho_{1}^{0}}{\partial t}+\operatorname{div}\left(\varrho_{1}^{0} v\right)\right]=0
$$

essendo $H_{\mathrm{o}}=U_{\mathrm{o}}+p$.

Poichè dalle relazioni

$$
\frac{\partial W^{\sigma}}{\partial x^{\sigma}}=\frac{\partial W^{\prime \sigma}}{\partial x^{\prime \sigma}} ; \quad d t^{\prime}=d t \sqrt{1-\beta^{2}}
$$


si deduce in prima approssimazione $\left(^{*}\right)$

$$
\operatorname{div} v \simeq \operatorname{div} v^{\prime} \simeq \operatorname{div} v_{1} ; \quad d t^{\prime} \simeq d t
$$

in virtù delle $(25),(33),(34)$ si può scrivere sensibilmente:

$$
\begin{aligned}
c^{2}\left[\frac{\partial \varrho_{1}^{0}}{\partial t}+\operatorname{div}\left(\varrho_{1}^{0} v\right)\right]= & c^{2} \\
& \left(\frac{d \varrho_{1}^{0}}{d t}+\varrho_{1}^{0} \operatorname{div} v\right) \simeq c^{2}\left(\frac{d \varrho_{1}^{0}}{d t^{\prime}}+\varrho_{\mathrm{I}}^{0} \operatorname{div} v^{\prime}\right)= \\
& =c^{2}\left[\frac{\partial \varrho_{1}^{0}}{\partial t^{\prime}}+\operatorname{div}\left(\varrho_{1}^{0} v_{1}\right)\right] \simeq-\operatorname{div}\left(\chi \operatorname{grad} T_{0}\right) \simeq-\operatorname{div}(\chi \operatorname{grad} T)
\end{aligned}
$$

essendo $T$ la temperatura del mezzo rispetto a $K$.

Pertanto, la (63) ci fornisce la seguente relazione energetica:

$$
\frac{\partial}{\partial t}\left(U_{c}+\frac{1}{2} \varrho^{*} v^{2}\right)+\operatorname{div}\left[\left(\frac{1}{2} \varrho^{*} v^{2}+H_{c}\right) v-\chi \operatorname{grad} T\right]=0
$$

che è stata stabilita direttamente ed in maniera rigorosa, anche nel caso più generale, nell'ambito classico $\left.{ }^{15}\right)$.

Tnoltre, essendo

$$
\varrho=\frac{h}{c^{2}} \simeq \varrho^{*}+\frac{1}{2} \varrho^{*} \beta^{2}+\frac{1}{c^{2}}\left(U_{\circ}+p\right) \simeq \varrho^{*},
$$

la $\left(66_{2}\right)$ si muta, in prima approssimazione, nella seguente equazione

$$
\frac{\partial}{\partial t}\left(\varrho^{*} v_{i}\right)+\frac{\partial}{\partial x^{k}}\left(p \delta_{i k}+\varrho^{*} v_{i} v_{k}\right)=0 .
$$

Com'è noto $\left({ }^{16}\right)$, le (72), (74) si deducono direttamente in fluidodinamica classica combinando le equazioni di Eulero, l'equazione di continuità ed i principi della termodinamica.

Il vettore:

$$
S=\left(\frac{1}{2} \varrho^{*} v^{2}+H_{\varepsilon}\right) v-\chi \operatorname{grad} T
$$

che compare nella (72), generalizza il vettore densità di corrente di energia fluidodinamica, introdotto da UMOV (17) nel 1874 relativamente al caso adiabatico.

$\left(^{*}\right)$ Con procedimento analogo a quello indicato nella nota a piè di pag. 9.

$\left({ }^{15}\right)$ G. CARInI, Atti Acc. Pelorit. dei Pericolanti, Cl. Sc. Fis. Mat. e Nat., 59 (1971), pp. 157-165 cfr. anche Atti IX Congresso U.M.I. (1971).

$\left({ }^{16}\right)$ Cfr. ad es. 1. cit. $\left({ }^{2}\right)$.

(17) N. A. UMov, The Equation of Motion for the Energy of a body, Odessa, 1874. 
Il tensore tridimensionale $\Pi_{i k}^{(0) 1}=p \delta_{i k}+\varrho^{*} v_{i} v_{k}$, che figura nella (74) caratterizza la corrente dell'impulso e notoriamente viene chiamato il "tensore densità di corrente dell'impulso ».

Nell'ambito classico, le (72), (74) regolano rispettivamente la corrente dell'energia fluidodinamica ed il flusso della quantità di moto rispetto a $K$.

L'interpretazione fisica delle (72), (74), testè accennata nell'ambito classico, continua a sussistere per le (66), valide in fluidodinamica relativistica con conduzione di calore.

In quest'ultimo caso, la $h$, che compare nella $\left(66_{1}\right)$, è la densità totale di energia relativistica del fluido rispetto a $K$; $h v$ è il vettore densità di corrente di energia, corrispondente al vettore di Umov della fluidodinamica classica.

Il tensore tridimensionale

$$
\Pi_{i k}=p \delta_{i k}+\varrho v_{i} v_{k}
$$

che interviene nella $\left(66_{2}\right)$ generalizza l'analogo tensore $\Pi_{i z}^{(\text {lal }}$ della fluidodinamica classica e caratterizza, nell'ambito relativistico, la corrente dell'impulso totale del mezzo rispetto a $K$.

6. - Osserviamo infine che le (66), provenienti dalle (48), sono compendiate nelle seguenti equazioni quadridimensionali:

$$
\frac{\partial T^{\sigma v}}{\partial x^{v}} \equiv T_{/ v}^{\sigma v}=0
$$

essendo

$$
T^{\sigma \nu}=c^{2} \varrho_{0} W^{\sigma} W^{\nu}-p g^{\alpha \nu}=H_{0} W^{\sigma} W^{\nu}-p g^{\alpha \nu}
$$

il tensore energetico del mezzo nel caso in esame.

Le (77) si deducono agevolmente dalle (48) trasformando i primi membri di queste ultime nel modo seguente:

$$
\frac{d}{d s}\left(d \bar{m}_{0} W^{\sigma}\right)=\frac{d}{d s}\left(\varrho_{0} d \nabla_{0} W^{\sigma}\right)=\frac{\partial}{\partial x^{\nu}}\left(\varrho_{0} W^{\sigma}\right) W^{\nu} d V_{0}+\varrho_{0} W^{\sigma} \frac{d}{d s}\left(d V_{0}\right)
$$

che, in virtù della (50), diventano

$$
\frac{d}{d s}\left(d \bar{m}_{0} W^{\sigma}\right)=\frac{\partial}{\partial x^{p}}\left(\varrho_{0} W^{\sigma} W^{v}\right) d V_{0} .
$$

Se ora si osserva che si ha

$$
g^{\sigma v} \frac{\partial p}{\partial x^{p}}=\frac{\partial}{\partial x^{\nu}}\left(p g^{a \nu}\right)
$$

dalle (48) si deducono immediatamente le (77). 
Pertanto concludiamo osservando che le (77), come le (66), regolano il flusso dell'energia relativistica ed il flusso della quantità di moto relativistica del mezzo considerato rispetto al riferimento $K$.

Da quanto precede si desume, d'accordo con la visione fisica di Landau-Lifschitz, che solo per quanto riguarda la struttura il tensore energetico di un fluido ideale termoconduttore è identico con quello di un fluido ideale adiabatico.

In realtà, nel primo caso, la densità di massa $\varrho$, come è stato ampiamente dimostrato nei numeri precedenti, implica la densità di massa proveniente dalla distribuzione della materia e quella dell'energia termica, espressa in termini di massa. 